\title{
Contest Design with Uncertain Performance and Costly Participation
}

\author{
Priel Levy and David Sarne \\ Department of Computer Science \\ Bar Ilan University, Israel \\ priel.levy@live.biu.ac.il, sarned@cs.biu.ac.il
}

\author{
Igor Rochlin \\ School of Computer Science \\ College of Management, Israel \\ igor.rochlin@gmail.com
}

\begin{abstract}
This paper studies the problem of designing contests for settings where a principal seeks to optimize the quality of the best contribution obtained, and potential contestants only strategize about whether to participate in the contest, as participation incurs some cost. This type of contest can be mapped to various real-life settings (e.g., selection of background actors based on headshots, photography contest). The paper provides a comparative game-theoretic based solution to two variants of the above underlying model: parallel and sequential contest, enabling a characterization of the equilibrium strategies in each. Special emphasis is placed on the case where the contestants are a priori homogeneous which is often the case in contests where ranking is mostly influenced by some probabilistic factors (e.g., luck) or whenever contestants are evaluated subjectively by a referee whose taste cannot be a priori predicted. Here, several (somehow counter-intuitive) properties of the equilibrium are proved, in particular for the sequential contest, leading to a comprehensive characterization of the principal's preference between the two.
\end{abstract}

\section{Introduction}

Contests have been used since the dawn of man as a mechanism for inducing individual efforts. In recent years, contests are used not only as a means for determining the best contestant, but also for generating value. For example DARPA is offering Grand Challenges to promote the development of cutting-edge technologies, scientific organizations run contests to enhance research (e.g., [Baarslag et al., 2013]), firms run contests to come up with new products (such as the LEGO Ideas contests [Schlagwein and Bjorn-Andersen, 2014]) and not-for-profit organizations are organizing contests for transformative solutions that benefit mankind (as with the Hult Prize (www . hultprize.org)).

The majority of contest research deals with models where contestants can influence their performance in the contest through the effort or money they put [Baik, 1994; Moldovanu and Sela, 2006]. In various settings, however, this is not the case as not only contestants have no control over their performance from the time of making their participation decision and onwards, but they are also uncertain concerning the way they will be evaluated in the contest. This holds whenever contestants are being evaluated subjectively by anonymous judges, based on the taste of the public (in case of a voting open to the public), based on criteria that are not fully disclosed to them, or simply based on luck or uncertain environment parameters (e.g., weather). For example, when an agency is posting on social media that it seeks background actors ("extras") no audition takes place. Instead, contestants submit their pre-made headshots, and the selection is made based on how applicants' headshots match some desired look (which can be completely subjective as it depends on the specific casting team member in charge). Similarly, at the time a department chair solicits submissions from faculty members for excellence in research prize, prospective candidates cannot influence anymore the quality of the results they submit (as the research has already been carried out and published) and the way their results will be evaluated by the department chair compared to others' is somehow uncertain. This recurs also in applications such as photos contests (as photographers submit the best photos they have shot and are uncertain about voters' tastes) and graduate students applying for a post-doc (as the evaluation of their achievements by the professor offering the position is uncertain and they cannot influence the substence of their achievements at the time they apply). Common to all the above examples, that the contestants' strategy is limited to participating or not participating in the contest. This latter decision becomes non-trivial whenever participation incurs some cost (e.g., locating and uploading photos, filling in registration details, sending a $\mathrm{CV}$, emotional suffer and reputational loss in case of not winning).

In this paper we study contests of the above type, i.e., the contestants' performance in the contest is beyond the control of contestants at the time of the contest and participation is costly. Unlike most prior literature, that was primarily focused on parallel contest models, in this work we consider also the option for a sequential contest. In sequential contest, only one contestant performs at a time and its performance measure is known to the following contestants. Such design is commonly used in real-life, e.g., in Olympic sports such as platform diving, pole vault and javelin throw. 
Contributions. The paper provides a comprehensive gametheoretic based analysis of the parallel and sequential contest model variants of contests where contestants only strategize about participation, specifying the conditions under which different kinds of equilibria hold. The analysis enables demonstrating that the preference of the contest to be used (parallel or sequential), from the contest organizer's point of view, can frequently change as a function of the setting parameters-even a slight variation in one of the parameters can result in several alternations in preference. For the case of homogeneous contestants, however, we manage to characterize some setting classes where parallel contest dominates a sequential one and vice versa. In particular, we prove a transition in preference from parallel to sequential contest as the ratio between the participation cost and the prize awarded increases. Interestingly (and somehow counter-intuitively), we find that in sequential contest with homogeneous contestants, despite the asymmetry imposed by the sequential process, in equilibrium both the contestant's strategies and their expected profit do not depend on the number of contestants nor the position of a contestant in the sequence. The latter finding suggests that the sequential competition is just as fair as the parallel one.

\section{Related Work}

Contests are organizational structures in which contestants spend costly efforts (e.g., time, resources) to win one or more prizes [Dechenaux et al., 2014]. Contest design, i.e., the set of rules that define a contest, had focused much interest in literature [Glazer and Hassin, 1988; Dasgupta and Nti, 1998; Ghosh and Kleinberg, 2016], differing primarily in the assumptions made in the underlying contest model (e.g., offering several prizes [Galton, 1902; Cohen et al., 2008; Archak and Sundararajan, 2009] or using more than a single stage (most commonly in the form of a tournament) [Rosen, 1986; Clark and Riis, 1996; 1998; Hazon et al., 2008]) and the contest organizer's goals (e.g., maximizing overall effort, best effort, fairness) [Lev et al., 2013]. In addition to foundational work on the theory of contests, much research has been devoted to providing experimental evidence for the way people behave in contests [Dechenaux et al., 2014] as well as online crowdsourcing contests [DiPalantino and Vojnovic, 2009; Chawla et al., 2012].

Most literature in the area of contest design deals with effort-based contests, where the effort expended by contestants determines their probability of winning a prize [Nti, 1999; Moldovanu and Sela, 2006] (perhaps the most common is the Tullock contest [Buchanan et al., 1983] in which the winning probability is the ratio of the contestant's effort and the total effort exerted by all contestants). Our model, as motivated above assumes contestants cannot influence their chance of winning at the time the contest takes place and their strategy space is limited to participating or opting not to participate in the contest. Among the few works that consider this kind of contest, the most relevant ones are Ghosh and Hummel (2012) and Ghosh and Kleinberg (2016). These works however are focused on the question of how to design a multi-prize scheme and are limited to the case of parallel contest (whereas ours considers also the option for a sequential one). More importantly, their model assumes contestants learn about their performance measure in the contest prior to having to decide on participation, whereas in our model there is no certainty concerning performance at the time the participation decision is made. This latter difference is fundamental, as it results in a completely different analysis (and solution structure).

Finally, our work differs from most existing contest-design work in a way that it considers the option of using a sequential contest and provides a comparative analysis of the sequential and parallel model variants. Prior work has focused primarily in simultaneous contest. The sequential contest is not completely absent in literature [Segev and Sela, 2014; Liu et al., 2014; Jian et al., 2016], however to the best of our knowledge has been analyzed only in the context of effort-based contest [Morgan, 2003; Fu and Lu, 2012; Stracke, 2013] with very little comparative analysis of the two.

\section{The Model}

The model considers a contest organizer and a set $A=$ $\left\{A_{1}, \ldots, A_{k}\right\}$ of $k>1$ heterogeneous potential contestants (denoted "agents" onwards). Agents are fully-rational and self-interested. Each agent $A_{i}$ can either participate in the contest, incurring some $\operatorname{cost} c_{i}$, or opt to avoid participating in the contest. The performance of an agent in the contest is a priori unknown and is being affected both by its inherent competence and various external factors (e.g., luck, weather conditions, refereeing). This is modeled by taking the performance of agent $A_{i}$ to be determined according to some probabilistic function $f_{i}(x)$ (where $F_{i}(x)$ is the corresponding cumulative distribution function).

The goal of the organizer is to maximize the expected maximum performance obtained by agents in a contest it runs. In order to encourage participation in the contest the organizer offers a prize $M>0$ to the agent ranked first (performancewise) in the contest. ${ }^{1}$ In case none of the agents choose to participate in the contest, the prize is randomly awarded to one of the agents and the performance as perceived by the organizer is set to some pre-set fallback performance $v_{0}{ }^{2}$ The choice of always awarding the prize is a standard modeling assumption in contest theory [Faravelli, 2011; Liu et al., 2013] and corresponds to the case where agents can opt to expend some default minimal performance (e.g., submitting a trivial code that chooses randomly in the Netflix challenge) without incurring any cost. The goal of each agent is to maximize its own expected profit, defined as the expected prize awarded to it minus the cost incurred if participating in the contest. It is assumed that $f_{i}(x), c_{i}$ and $M$ are all common knowledge in the sense that they are known to all agents and to the organizer [Moldovanu and Sela, 2001; 2006; Luo et al., 2014].

\footnotetext{
${ }^{1}$ Since performance is continuous, the chance of having two agents ranked first is negligible. Otherwise, a tie-breaking rule is required.

${ }^{2}$ As otherwise the expected maximum performance is undefined. Typically this will be zero.
} 
The analysis considers two model variants, differing in the way the contest is designed. The first is based on simultaneous participation ("parallel contest"), i.e., each agent's participation decision takes part in parallel to the others'. The second is based on sequential participation ("sequential contest"). Here, each agent in its turn (according to some pre-defined order) gets to see the results of its predecessors (whether participated, and if so also their performance) and then decides whether to participate in the contest.

\section{Analysis}

The analysis is divided according to the contest type (parallel and sequential).

\subsection{Parallel Contest}

We use $\{P, \neg P\}$ to denote the actions available to each agent, where $P$ stands for participate and $\neg P$ for not participate. Since the game in this case is simultaneous, every agent $A_{i}$ 's strategy can be captured by the probability $p_{i}\left(0 \leq p_{i} \leq 1\right)$ it chooses action $P\left(\forall A_{i} \in A\right)$.

Consider agent $A_{i}$. Given the strategy profile of all other agents $\left\{p_{j} \mid A_{j} \in A \wedge A_{j} \neq A_{i}\right\}$, the probability that the maximum performance obtained by others is equal to or less than $y$, denoted $\bar{F}_{i}(y)$, is:

$$
\bar{F}_{i}(y)=\prod_{A_{j} \in A-\left\{A_{i}\right\}}\left(p_{j} F_{j}(y)+\left(1-p_{j}\right)\right)
$$

The expected profit of agent $A_{i}$ if participating, denoted $B_{i}^{P}$, is thus given by:

$$
B_{i}^{P}=-c_{i}+M \int_{y=-\infty}^{\infty} f_{i}(y) \bar{F}_{i}(y) d y
$$

i.e., agent $A_{i}$ is awarded the prize $M$ whenever its performance $y$ is the maximum among all agents that participate.

Similarly, the expected profit of agent $A_{i}$ if not participating, denoted $B_{i}^{\neg}$, is given by:

$$
B_{i}^{\neg P}=\frac{M}{k} \prod_{A_{j} \in A-\left\{A_{i}\right\}}\left(1-p_{j}\right)
$$

i.e., with probability $\prod_{A_{j} \in A-\left\{A_{i}\right\}}\left(1-p_{j}\right)$ neither of the other $k-1$ agents participate and the prize $M$ is randomly awarded to one of the $k$ agents.

The best response strategy of every agent $A_{i}$ is thus to participate if $B_{i}^{P}>B_{i}^{P}$ and not participate otherwise. A Bayesian Nash Equilibrium (BNE) solution $\left\{p_{1}, \ldots, p_{k}\right\}$ to the parallel contest should therefore satisfy: (a) for every agent $A_{i}$ for which $p_{i}=0, B_{i}^{P} \leq B_{i}^{\neg}$; (b) for every agent $A_{i}$ for which $p_{i}=1, B_{i}^{P} \geq B_{i}^{\neg}$; and (c) for every agent $A_{i}$ for which $0<p_{i}<1, B_{i}^{P}=B_{i}^{\neg P}$. It is possible that a given setting will have more than a single equilibrium solution (i.e., multi-equilibria), though the question of which of those will be used is beyond the scope of the current paper.

One specific setting where we can determine the nature of equilibrium in terms of the strategies to be used is where the agents differ only in their participation costs (e.g., in the background actors search where participants differ primarily in their costs of locating and uploading photos) as stated in the following proposition.
Proposition 1. When $f_{i}(x)=f_{j}(x) \forall i, j, x$, the BNE obtained is: (a) strictly in pure strategies such that all agents participate, whenever $\frac{c_{i}}{M} \leq \frac{1}{k} \forall i$; (b) strictly in pure strategies such that all agents do not participate, whenever $\frac{c_{i}}{M} \geq$ $\frac{k-1}{k} \forall i$; and $(c)$ in mixed or pure strategies, where different agents use different strategies, otherwise.

Proof. For case (a), since $f_{i}(x)=f_{j}(x) \forall i, j$, all participants in the contest are equally likely to win. Therefore participation results in an expected profit of at least $\frac{M}{k}-c_{i} \geq 0$ and hence it is the best response for the agent if the number of other participants is at least one (in which case not participating results in a zero profit). If no other agent participates then if agent $A_{i}$ participates its profit is $M-c_{i}$ and otherwise $\frac{M}{k}$. The difference between the two is: $M-c_{i}-\frac{M}{k}=$ $\frac{M(k-1)}{k}-c_{i} \geq c_{i}(k-1)-c_{i} \geq 0$. Hence even in this case participation is the best strategy. For case (b), any individual agent $A_{i}$ has an incentive not to participate, regardless of the strategy used by the others, as otherwise its expected profit is bounded from above either by $M-c_{i} \leq \frac{M}{k}$ in case no one else participates, or by $\frac{M}{k}-c_{i}<\frac{c_{i}}{k-1}-c_{i} \leq 0$ in case the number of the other participants is at least one $(k>1)$. In all other cases, there is an incentive for at least one agent to participate in the contest if all others opt not to participate, and similarly to opt not to participate if all others do participate.

Finally, the organizer's expected profit is given by:

$$
B^{\text {org }}=\int_{y=-\infty}^{\infty} \max \left(y, v_{0}\right) \frac{d(\bar{F}(y))}{d y} d y
$$

where $\bar{F}(y)=\prod_{A_{j} \in A}\left(p_{j} F_{j}(y)+\left(1-p_{j}\right)\right)$ is the probability that the maximum performance obtained in a contest involving $k$ agents is less than $y$.

\subsection{Sequential Contest}

In the case of a sequential contest the (subgame perfect) BNE is fully in pure strategies, as agents have perfect information about the performance of preceding agents. An agent's strategy $S$ is its choice of participation given the best performance obtained so far, formally captured by the function $S: \mathbb{R} \cup \emptyset \rightarrow\{P, \neg P\}$, where $\emptyset$ is the case where all former agents opted not to participate in the contest. For exposition purposes we align the agents' participation order with their index.

Consider an agent $A_{i}$ when the best performance obtained so far is $v$ and the strategies used by all other agents are given by $S_{-i}=\left\{S_{1}, \ldots, S_{i-1}, S_{i+1}, . ., S_{k}\right\}$. We use the function $F_{i}^{\text {next }}(y)$ to denote the probability that given that the best performance reached once $A_{i}$ participates is $y$ the maximum performance obtained by all agents participating after $A_{i}$ is equal to or less than $y$. The expected profit of $A_{i}$ if participating, whenever receiving $v$, denoted $B_{i}^{P}(v)$, is thus:

$$
B_{i}^{P}(v)= \begin{cases}M \int_{y=v}^{\infty} f_{i}(y) F_{i}^{n e x t}(y) d y-c_{i} & v \neq \emptyset \\ M \int_{y=-\infty}^{\infty} f_{i}(y) F_{i}^{n e x t}(y) d y-c_{i} & v=\emptyset\end{cases}
$$


Similarly, the expected profit of $A_{i}$ if not participating, whenever receiving $v$, denoted $B_{i}^{\neg P}(v)$, is given by:

$$
B_{i}^{\neg P}(v)= \begin{cases}\frac{M}{k} & v=\emptyset \wedge S_{j}(\emptyset)=\neg P \forall j>i \\ 0 & \text { otherwise }\end{cases}
$$

Theorem 1. The best-response strategy of any agent $A_{i}$ given the strategies of the other agents $S_{-i}$ and the best performance obtained so far $v$ is: (a) use a threshold $r_{i}$ to determine whether or not to participate in case $v \neq \emptyset$ and there is $r_{i}$ satisfying:

$$
\frac{c_{i}}{M}=\int_{y=r_{i}}^{\infty} f_{i}(y) F_{i}^{n e x t}(y) d y
$$

where:

$$
F_{i}^{\text {next }}(y)= \begin{cases}\prod_{\left(S_{j}(y)=P\right) \wedge(j>i)} F_{j}(y) & i \leq k \\ 1 & i=k\end{cases}
$$

(b) not participate in case $v \neq \emptyset$ and there is no $r_{i}$ satisfying (6); (c) participate if $\frac{k-1}{k} \geq \frac{c_{i}}{M}$ and otherwise not participate, in case $v=\emptyset$ and $S_{j}(\emptyset) \stackrel{M}{=} \neg P \forall j>i$; (d) participate if $\frac{c_{i}}{M} \leq \int_{y=-\infty}^{\infty} f_{i}(y) F_{i}^{\text {next }}(y) d y$ and otherwise not participate, in case $v=\emptyset$ and $\exists j>i$ such that $S_{j}(\emptyset)=P$.

Proof. For the case where $v \neq \emptyset$, participating is better than not participating if $M \int_{y=v}^{\infty} f_{i}(y) F_{i}^{n e x t}(y) d y-c_{i} \geq 0$ (according to (4) and (5)). Since $\int_{y=v}^{\infty} f_{i}(y) F_{i}^{\text {next }}(y) d y$ decreases in $v$, participating is the preferred choice for any performance $v$ that is lesser than $r_{i}$ for which (6) holds. This proves part (a). If there is no $r_{i}$ satisfying (6) then it can either be because $\int_{y=r_{i}}^{\infty} f_{i}(y) F_{i}^{\text {next }}(y) d y$ is always greater than $\frac{c_{i}}{M}$ or always lesser than $\frac{c_{i}}{M}$. However, the term reaches zero for $r_{i} \rightarrow \infty$ and therefore it is necessarily the case where the expected gain from participating is lower than the cost incurred, resulting in preferring not to participate. This proves part (b).

The case of $v=\emptyset$ is different as the agent can potentially gain from not participating if no one else will choose to participate as well. According to (4) and (5), in case $S_{j}(\emptyset)=\neg P$ $\forall j>i$, participating is the preferred choice if $M-c_{i} \geq \frac{M}{k}$ and therefore the distinction between the two cases in part (c). Finally, when $v=\emptyset$ and $\exists j>i$ such that $S_{j}(\emptyset)=P$, the decision is based solely on the chance of winning, being the first to participate. The proof in this case is similar to the one provided for case (a), in the sense of equating gains and costs incurred, and therefore omitted.

A BNE is thus of the form $\left(\left(C_{1}^{\emptyset}, C_{1}^{\neg \emptyset}\right), \ldots,\left(C_{k}^{\emptyset}, C_{k}^{\neg \emptyset}\right)\right)$, where $C_{i}^{\emptyset} \in\{P, \neg P\}$ corresponds to the case of being reached with a performance $\emptyset$ and $C_{i}^{\neg \emptyset} \in\left\{P, \neg P, r_{i}\right\}$ corresponds to the case of being reached with some real performance $v$, satisfying the conditions specified in Theorem 1 .

We now turn to calculating the expected profit of the organizer. Let $B_{i}^{\text {org }}(v)$ denote the organizer's expected profit when the current participant is $A_{i}$ (with $k-i+1$ more agents to go) and the maximum performance of the preceding $i-1$ agents is $v$. The value $B_{i}^{o r g}(v)$ is calculated based on $A_{i}$ 's position in the sequence. If $A_{i}$ is the last agent (i.e., $i=k)$ and does not participate $\left(S_{i}(v)=\neg P\right)$ the organizer obtains the maximum between the default profit $v_{0}$ and the performance $v$ (or trivially $v_{0}$ if $v=\emptyset$ ). In case $A_{i}$ does participate $\left(S_{i}(v)=P\right)$ the profit relies on the maximum between $v_{0}$ and the performance obtained by $A_{i}: B_{i}^{\text {org }}(v)=\int_{y=-\infty}^{\infty} \max \left(y, v_{0}\right) f_{i}(y) d y$ if $v=\emptyset$ and $B_{i}^{\text {org }}(v)=\int_{y=v}^{\infty} \max \left(y, v_{0}\right) f_{i}(y) d y+F_{i}(v) \max \left(v, v_{0}\right)$ otherwise. If $A_{i}$ is not the last in the sequence $(i<k)$ then we replace the actual performances $y$ and $v$ in the above by the expected profits $B_{i+1}^{\text {org }}(y)$ and $B_{i+1}^{\text {org }}(v)$, as the contest is to be continued with the transition to agent $A_{i+1}$, resulting in: $B_{i}^{\text {org }}(v)=B_{i+1}^{\text {org }}(v)$ for $S_{i}(v)=\neg P, B_{i}^{\text {org }}(v)=$ $\int_{y=-\infty}^{\infty} f_{i}(y) B_{i+1}^{o r g}(y) d y$ for $S_{i}(v)=P$ and $v=\emptyset$, and $B_{i}^{\text {org }}(v)=\int_{y=v}^{\infty} f_{i}(y) B_{i+1}^{\text {org }}(y) d y+F_{i}(v) B_{i+1}^{\text {org }}(v)$ otherwise. The organizer's expected profit from the contest, denoted $B^{\text {org }}$, is given by $B^{\text {org }}=B_{1}^{\text {org }}(\emptyset)$.

\section{Influencing Dynamics}

The choice of the type of contest (sequential or parallel) to be used by the organizer is not trivial and the preference of type may alternate even with the slightest change in setting parameters. In this section we illustrate this by following the changes in the nature and structure of the equilibrium obtained, and consequently the organizer's preference, as the participation cost of one of the agents changes. The setting used includes three agents, where $c_{1}=c_{2}=0.16$ and $c_{3}$ is the independent parameter. All three agents are characterized by a uniform performance distribution function between 0 and 1 (i.e., $f_{1}(x)=f_{2}(x)=f_{3}(x)=1$ for $0 \leq x \leq 1$ and zero otherwise). The prize to be awarded to the winner is $M=0.4$ and the fallback performance is $v_{0}=0$.

Figure 1 compares the organizer's expected profit in the above setting when using sequential and parallel contest, as a function of the participation cost $c_{3}$ of $A_{3}$ (Graph (a)). Graphs (b) and (c) depict the strategies used by the different agents in the equilibrium obtained, enabling a better understanding of the behaviors reflected in the organizer's expected profit. For exposition purposes we use $\left(p_{1}, p_{2}, p_{3}\right)$ to denote the equilibrium in the parallel case, where $p_{i}$ is the participation probability of agent $A_{i}$. For the sequential case we present the participation's thresholds $r_{2}$ and $r_{3}$.

We begin with the parallel contest. Here, there are several equilibria, and the curve used depicts the one in which $A_{1}$ and $A_{2}$ are using a symmetric strategy. This latter choice is made for two primary reasons: First, since $A_{1}$ and $A_{2}$ are symmetric, the most natural (and fair) equilibrium is the one where they use the same strategy (and gain the same profit). Second, in this example, the symmetric equilibrium is the one that maximizes the organizer's expected profit. For $c_{3}<M / 3=$ 0.133 we obtain an equilibrium $(0.6,0.6,1)$. Other equilibria are $(1,0,1)$ and $(0,1,1)$. In the interval $0.133<c_{3}<0.186$ a mixed equilibrium of type $\left(p, p, p_{3}\right)$, which could not hold for $c_{3}<M / 3$, replaces the $(p, p, 1)$ solution which is not stable anymore. Interestingly, in the transition that takes place at $c_{3}=0.133$ we observe another counter-intuitive phenomena according to which a decrease in the competence of one of the 


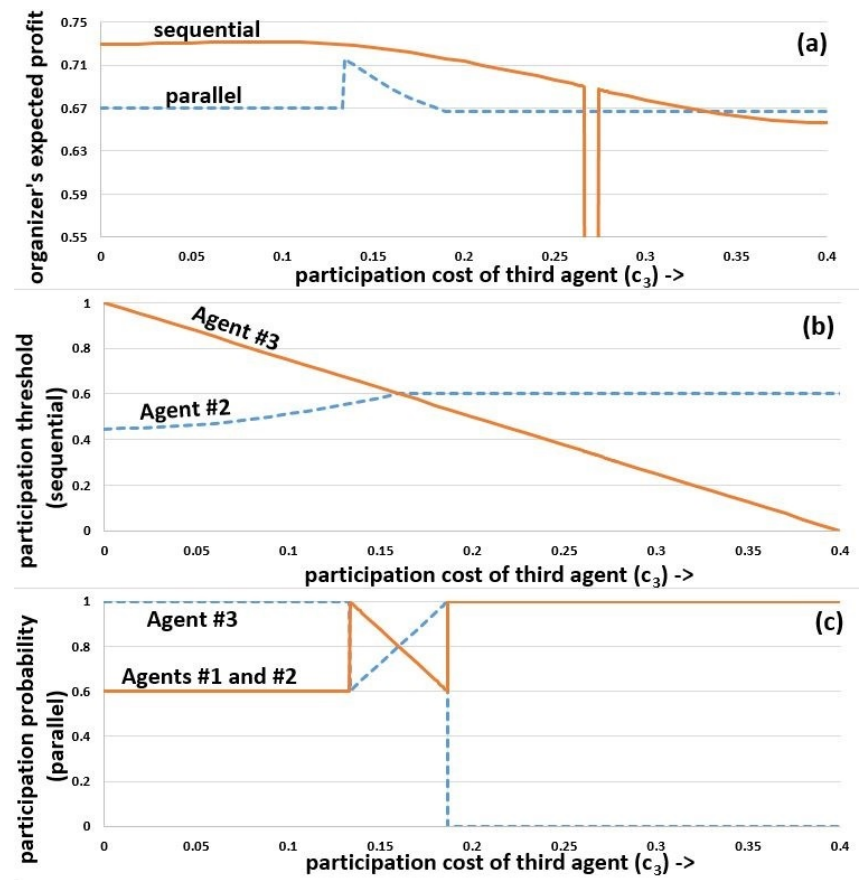

Figure 1: The influence of $c_{3}$ over: (a) organizer's expected profit; (b) participation thresholds when competing sequentially; and (c) participation probabilities in a parallel contest. See main text for the details of the setting used.

agents (an increase in the participation cost of $A_{3}$ in this case) results in an increase in the organizer's expected profit. In the interval $0.186<c_{3} \leq M / 2=0.2$ there are three equilibria, each characterized by having two of the agents participate in the contest and the third not participating, all resulting in an expected profit of $B^{\text {org }}=2 / 3$. Finally, with $c_{3}>0.2$ we obtain the single equilibrium $(1,1,0)$, resulting once again in $B^{\text {org }}=2 / 3$.

With the sequential contest, we obtain a single BNE. As expected, the threshold used by $A_{3}$ decreases as its participation cost increases. The decreasing competition from $A_{3}$ results in an increase in the threshold used by $A_{2}$. For $c_{3}=0.16$ all three agents are homogeneous and indeed $r_{2}=r_{3}=0.6$ as calculated by (11) which determines the threshold used with homogeneous agents. Once $r_{3}$ becomes less than 0.6 , the threshold used by $A_{2}$ becomes fixed and equals 0.6 . This can intuitively be explained by the fact that in the absence of $A_{3}$ the threshold used by $A_{2}$ is $r_{2}=0.6$ (according to (11)). Meaning that $A_{2}$ 's expected profit in those cases where its performance is greater than 0.6 is enough to justify participation. However adding $A_{3}$ with a strategy $r_{3}<0.6$ does not affect $A_{2}$ 's profit whenever performing better than 0.6 , as $A_{3}$ opts not to participate in such cases.

Finally, when $A_{3}$ 's participation cost becomes big enough ( $\left.c_{3}=2 M / 3=0.267\right)$, the BNE's nature changes to having all three agents opt not to participate (in which case the organizer's expected profit drops to zero). This is because as long as $A_{1}$ and $A_{2}$ do not participate, for any $c_{3}>M-\frac{M}{3}$ not participating dominates participating for $A_{3}$. In such case, if $A_{1}$ is not participating then $A_{2}$ gains more from not participat- ing and receiving $\frac{M}{3}$ than participating (and hence invoking also $A_{3}$ 's participation, though with a relatively small threshold $r_{3}$ ). Similar considerations apply to $A_{1}$. Interestingly, for $c_{3}>0.273$ these considerations do not hold anymore, as $A_{3}$ becomes so incompetent that $A_{2}$ actually finds it beneficial to deviate towards participating even when $A_{1}$ does not participate.

As can be seen in Graph (a) of the figure, there are three transition points in the organizer's preference of the type of contest to be used. Further transitions in preference occur with different orderings of the agents in the sequential contest model, as well as changes in $c_{1}, c_{2}$ and $M$.

\section{Homogeneous Agents}

In many real-life contest settings agents are homogeneous in the sense that they share the same participation cost $\left(c_{i}=c\right.$, $\forall i$ ) and the probability distribution function according to which their performance is determined $\left(f_{i}(x)=f(x), \forall i\right)$. Meaning that the agents are a priori homogeneous-even though each of them will end up with a different performance at the contest, at the time they make their participation decision neither of them has an a priori advantage. ${ }^{3}$ The analysis of the homogeneous case reveals several interesting properties related to the structure of the game equilibrium.

\subsection{Parallel Contest}

Since the agents are homogeneous we are interested in the symmetric equilibrium (i.e., $p_{i}=p \forall i$ ), as it is the natural and fair one. When using the same $p$, Equations (1) and (2) can be substantially simplified and expressed as follows:

$$
\begin{gathered}
B_{i}^{P}=\sum_{j=0}^{k-1}\left(\begin{array}{c}
k-1 \\
j
\end{array}\right) \frac{1}{j+1} M p^{j}(1-p)^{k-j-1}-c \\
B_{i}^{\neg P}=\frac{M}{k}(1-p)^{k-1}
\end{gathered}
$$

Theorem 2 provides a comprehensive characterization of the $\mathrm{BNE}$ in this case, as a function of the ratio $\frac{c}{M}$, including a closed form solution for $p$.

Theorem 2. In the homogeneous parallel contest case, the BNE is: (a) fully based on pure strategies such that $p=1$ (all agents participate) and $p=0$ (all agents opt not to participate) when $\frac{c}{M} \leq \frac{1}{k}$ and $\frac{c}{M} \geq \frac{k-1}{k}$, respectively; (b) based on mixed strategies, otherwise, where $p$ is the solution to:

$$
\frac{c}{M}=\frac{1-(1-p)^{k-1}}{k p}
$$

Proof. Part (a) directly derives from Proposition 1. In any other case there is necessarily one equilibrium which is based on mixed strategies that use the same $p$. Equating (8) to (9) results in $\frac{c}{M}=\sum_{j=1}^{k-1}\left(\begin{array}{c}k-1 \\ j\end{array}\right) \frac{1}{j+1} p^{j}(1-p)^{k-j-1}+\frac{k-1}{k}(1-$ $p)^{k-1}$. Using the identity $\left(\begin{array}{c}k \\ j+1\end{array}\right)=\frac{k}{j+1}\left(\begin{array}{c}k-1 \\ j\end{array}\right)$ and the binomial theorem we obtain (10).

${ }^{3}$ This is also the case in many of the applications motivating this research (e.g., in the background actors search where in the absence of concrete guidelines contestants are typically a priori alike, or in the photography contests where submissions are typically of high quality and the public taste is difficult to predict). 
The derivative of the right-hand-side of (10) with respect to $p$ is always negative, hence the value of $p$ (and consequently the expected profit of the organizer) increases as participation cost $c$ decreases over the interval $\frac{1}{k}<\frac{c}{M}<\frac{k-1}{k}$.

\subsection{Sequential Contest}

Theorems 3 and 4 provide a comprehensive characterization of the BNE for the case of sequential contest with homogeneous agents as a function of the problem parameters.

Theorem 3. The equilibrium solution to the homogeneous case is: (a) have all agents not participate whenever $\frac{c}{M} \geq$ $\frac{k-1}{k} ;(b)$ have all agents participate whenever $c=0 ;(c)$ otherwise, have all agents use the same threshold $r$ to determine whether or not to participate, where r satisfies:

$$
\frac{c}{M}=\int_{y=r}^{\infty} f(y) d y=(1-F(r))
$$

Proof. The proof for the case where $\frac{c}{M} \geq \frac{k-1}{k}$ is a trivial modification of the proof given for case (b) of Proposition 1. The case where $c=0$ is straightforward-if not incurring a participation cost then participating dominates not participating. Therefore we only need to prove the part where $0<\frac{c}{M}<\frac{k-1}{k}$. This is proved by induction: The last agent $A_{k}$ uses $r$ according to (11) based on substituting $F_{i}^{n e x t}(y)=1$ (according to (7)) in (6). Now assume that every agent $A_{j}$ for which $j>i$ uses $r_{j}=r$ according to (11). We prove that $r_{i}=r$ as well. Assume otherwise, e.g., $r_{i}<r$. Now consider any value $z$ such that $r_{i}<z<r$. Since $z>r_{i}$, not participating dominates participating if being reached with a value $z$ (i.e., $B_{i}^{P}(z)<0$ ). However according to the proof's assumption all remaining agents use $r$ and therefore the expected profit of the agent if participating is: $B_{i}^{P}(z)=-c+M\left(\int_{y=r_{i}}^{r} f(y)(F(y))^{k-i} d y+(1-F(r))\right)$. Substituting $c=M(1-F(r))$ (according to (11)) in the latter term obtains $B_{i}^{P}(z)=M \int_{y=r_{i}}^{r} f(y)(F(y))^{k-i} d y>0$ which is a contradiction. Similarly, assume $r_{i}>r$ and a value $z$ such that $r<z<r_{i}$. Since $z<r_{i}$ participating dominates not participating if being reached with a value $z$ (i.e., $B_{i}^{P}(z)>0$ ). However if participating in the contest the expected profit of the agent is: $B_{i}^{P}(z)=-c+M(1-F(z))<$ $-c+M(1-F(r))=0$, which leads again to contradiction.

The use of a threshold-based strategy and in particular the fact that the exact same threshold is used by all agents can be explained through similar intuitions that hold in optimal stopping problems such as those studied by McMillan and Rothschild [McMillan and Rothschild, 1994]. Here a searcher needs to decide when to stop (and exploit the current value observed) when sampling repeatedly from a distribution while the sampling is costly. Despite some inherent differences (most observable is the fact that in our model each decision is made by a different agent in a competition context) the underlying process is somehow similar in its nature, and indeed the optimal strategy for the optimal stopping problem is known to be threshold-based and uses the same threshold over each sampling period.
Theorem 4. In equilibrium, all agents gain the exact same expected profit $B$, regardless of their position in the sequence, depending on the nature of the equilibrium: (a) $\frac{M}{k}$ if the equilibrium strategy is not to participate; (b) $\frac{M}{k}-c$ if they all participate (i.e., when $c=0$ ); and $(c)$ otherwise:

$$
B=\frac{M(F(r))^{k}}{k}
$$

Proof. The first two cases are trivial and result from the model definition. As for the third case, the expected profit $B_{i}$ of agent $A_{i}$ is given by:

$B_{i}=\int_{y=-\infty}^{r}(i-1) f(y)(F(y))^{i-2}\left(M \int_{z=y}^{r} f(z)(F(z))^{k-i} d z+M \int_{z=r}^{\infty} f(z) d z-c\right) d y$

where $(i-1) f(y)(F(y))^{i-2}$ is the probability distribution function of getting to $A_{i}$ with a performance $y$ and $(F(z))^{k-i}$ is the probability that the remaining $k-i$ agents will fail to perform better than $y$. Substituting $\int_{y=r}^{\infty} f(y) d y=\frac{c}{M}$ (according to (11)) and further applying several standard mathematical manipulations we obtain (12).

The implications of Theorems 3 and 4 are quite surprising and counter-intuitive: with homogeneous agents even though each agent has full information concerning the performance of those participating before it, in equilibrium all agents follow the same participation rule and that rule does not depend on the number of participants $k$ (whenever choosing to participate) or the number of remaining agents. Furthermore, despite the fact that the chance of each agent to participate decreases (as it depends on the chance that preceding agents performed worse than $r$ ), along the agents sequence, all agents end up with the same expected profit, regardless of their position in the contest sequence.

Notice that from (11) we obtain that $F(r)=\frac{M-c}{M}$ and substituting it in (12) yields:

$$
B=\frac{(M-c)^{k}}{k M^{k-1}}
$$

meaning that the expected profit of any of the agents can be calculated even without calculating first the threshold-based strategy. Furthermore, $\frac{(M-c)^{k}}{k M^{k-1}}<\frac{M}{k}$, meaning that the agents are better off having all of them not participate in the contest. Unfortunately, this latter solution is not stable, leading as in many other problems to the tragedy of the commons.

If none of the agents participate the organizer's expected profit is $v_{0}$. Otherwise, her expected profit is given by (3), where:

$$
\bar{F}(y)= \begin{cases}(F(y))^{k} & y<r \\ (F(r))^{k}+\frac{1-(F(r))^{k}}{1-F(r)}(F(y)-F(r)) & y \geq r\end{cases}
$$

In case $y<r$ all agents obtain a value below $y$. In case $y \geq r$ there are two possible scenarios. The first is where all $k$ agents obtain a value below their threshold $r$, i.e., with probability $F(r)^{k}$. The second is where the contest terminates right after $j$ agents, upon revealing a value $z$ such that $r<z<y$ (otherwise, if $z<r$ the agents would decide to participate) and all former $j-1$ agents obtained a value smaller than $r$. The probability of the latter case occurring (summing over all values of $j \leq k$ ) can be calculated using the geometric series $\sum_{j=1}^{k}(F(y)-F(r)) F(r)^{j-1}$. 


\subsection{Methods Comparison}

The analysis provided in the former section unfolds several inherent differences between the parallel and sequential contest in the homogeneous case. The most significant difference is the dependency of the equilibrium strategy on the underlying distribution function $f(x)$ and the number of agents. From Theorem 2 we obtain that the parallel contest equilibrium strategies depend on the number of agents $k$ however do not depend whatsoever on $f(x)$. Intuitively, this results from the fact that the competing agents, being a priori homogeneous, are all equally likely to win. With the sequential contest we obtain the reversed phenomenon - the probability distribution function plays a key role in shaping the agents' equilibrium strategies while the number of agents has no effect at all (see Theorem 3). The independence of the agents' equilibrium strategies in the number of participating agents can once again be explained by similar intuitions as those given in optimal stopping problems for the independence of the optimal stopping strategy in the number of sampling periods available for the searcher.

From the agents' point of view, in both methods the expected profit does not depend on the underlying performance distribution. In the parallel model variant this derives from Theorem 2 according to which neither the segments defining the different equilibria types and the $p$ used (in case (b), according to (10)) do not depend on the distribution function. In the sequential case, this derives from (13).

In the homogeneous case, we can identify some conditions for determining the type of contest that will maximize the organizer's expected profit. These are captured by Proposition 2.

Proposition 2. From the organizer's point of view (i.e., $B^{\text {org }}$ wise): (a) when $c=0$ or $\frac{c}{M} \geq \frac{k-1}{k}$ both the parallel and sequential contests result in the same expected profit; $(b)$ when $0<\frac{c}{M} \leq \frac{1}{k}$ the parallel contest dominates the sequential one; and (c) for $\frac{1}{k}<\frac{c}{M}<\frac{k-1}{k}$ there exists at least one alternation point where the preference shifts from parallel to sequential contest.

Proof. In case $c=0$ all agents participate in the contest, as proved in Theorems 2 and 3. Therefore the expected profit is identical. Similarly, in the case $\frac{c}{M} \geq \frac{k-1}{k}$ all agents opt not to participate in the contest (Theorems 2 and 3 ) hence the expected profit is zero regardless of the method used. When $0<\frac{c}{M} \leq \frac{1}{k}$ all agents participate in a parallel contest (Theorem 2), while with a sequential contest it is guaranteed that only the first agent participates, however the participation of the others depend on the performance of their predecessors. Therefore the parallel contest yields a greater expected profit.

For $\frac{1}{k}<\frac{c}{M}<\frac{k-1}{k}$, we already know (proved above) that when $\frac{c}{M}=\frac{1}{k}$ parallel contest dominates sequential contest, hence to prove that there is an alternation point we need to show that for $\frac{c}{M} \rightarrow \frac{k-1}{k}$ the sequential contest dominates the parallel. Substituting $\frac{c}{M} \rightarrow \frac{k-1}{k}$ in (8) and (9) and equating the two (to obtain the mixed strategy $p$ ) obtains:

$$
\frac{k-1}{k}=\sum_{j=1}^{k-1}\left(\begin{array}{c}
k-1 \\
j
\end{array}\right) \frac{1}{j+1} p^{j}(1-p)^{k-j-1}+\frac{k-1}{k}(1-p)^{k-1}
$$

The above can hold only when $p \rightarrow 0$. Therefore, the expected profit is $B^{\text {org }} \rightarrow 0$. With sequential contest, on the other hand, when $\frac{c}{M} \rightarrow \frac{k-1}{k}$ we obtain $r \rightarrow F^{-1}\left(\frac{1}{k}\right)$ (according to (11)), hence there will be at least one participant, leading to an expected profit $B^{\text {org }}>0$.

Surprisingly, with parallel contest, the effect of the increase in the number of agents over the organizer's expected profit is not that clear - while one would expect agents to become more reluctant to participate due to the increased competition, the actual result can be either an increase or decrease in $p$. To illustrate, consider the case where $M=0.4, v_{\emptyset}=0$ and a uniform distribution function over the interval $(0,1)$. Here, if $c=0.15$ then in the transition from $k=3$ to $k=4$ the value of $p$ decreases from 0.875 to 0.634 , and consequently the organizer's expected profit decreases from 0.714 to 0.687 , while in the case of $c=0.25$ the value of $p$ increases from 0.125 to 0.177 and consequently the organizer's expected profit increases from 0.172 to 0.297 . This cannot happen in the sequential contest (proof is omitted for space considerations).

\section{Discussion and Conclusions}

As demonstrated numerically, the preference of the model to be used highly varies in the setting parameters, in the general case. This is where the analysis provided in the paper becomes particularly important, as it enables a contest organizer with the appropriate mathematical tools for extracting the equilibrium in both model variants. While the model assumes (much like most existing literature) the prize is always awarded, the transition to a model where the prize is not awarded if none of the agents participates is straightforward and requires only changing the profit in case of not participating $\left(B^{\neg}\right)$ to zero. Still, in this latter variant one has to consider a multi-attribute utility function for the organizer, to reflect the tradeoff between an increase in the performance achieved and the prize payments made.

The analysis of the homogeneous case unfolds several interesting properties of the equilibrium in the two models, many of which are highly counter-intuitive (especially in the case of sequential contest). In particular, an important implication of Theorem 4 is that an agent's profit does not depend on its position in the sequence of participants. This has many important social aspects from the contest design point of view, as it completely eliminates the need to compensate agents for their position in the sequence in order to maintain fairness.

We see many directions for future research extending this work. Among these are multi-prize allocation for enhancing participation, sequencing algorithms for maximizing profit in the case of sequential contest with heterogeneous agents and prize-setting algorithms for maximizing social welfare.

\section{Acknowledgments}

This research was partially supported by the ISRAEL SCIENCE FOUNDATION (grants No. 1083/13) and the ISFNSFC joint research program (grant No. 2240/15). 


\section{References}

[Archak and Sundararajan, 2009] Nikolay Archak and Arun Sundararajan. Optimal design of crowdsourcing contests. ICIS 2009 proceedings, page 200, 2009.

[Baarslag et al., 2013] Tim Baarslag, Katsuhide Fujita, Enrico $\mathrm{H}$ Gerding, Koen Hindriks, Takayuki Ito, Nicholas $\mathrm{R}$ Jennings, Catholijn Jonker, Sarit Kraus, Raz Lin, Valentin Robu, et al. Evaluating practical negotiating agents: Results and analysis of the 2011 international competition. Artificial Intelligence, 198:73-103, 2013.

[Baik, 1994] Kyung Hwan Baik. Effort levels in contests with two asymmetric players. Southern Economic Journal, 61(2):367-378, 1994.

[Buchanan et al., 1983] James M. Buchanan, Robert D. Tollison, and Gordon Tullock. Toward a theory of the rentseeking society. Public Choice, 41(2):339-345, 1983.

[Chawla et al., 2012] Shuchi Chawla, Jason D. Hartline, and Balasubramanian Sivan. Optimal crowdsourcing contests. In Proc. of SODA, pages 856-868, 2012.

[Clark and Riis, 1996] Derek J. Clark and Christian Riis. A multi-winner nested rent-seeking contest. Public Choice, 87(1):177-184, 1996.

[Clark and Riis, 1998] Derek J. Clark and Christian Riis. Influence and the discretionary allocation of several prizes. European J. of Political Economy, 14(4):605-625, 1998.

[Cohen et al., 2008] Chen Cohen, Todd Kaplan, and Aner Sela. Optimal rewards in contests. RAND Journal of Economics, 39(2):434-451, 2008.

[Dasgupta and Nti, 1998] Ani Dasgupta and Kofi O. Nti. Designing an optimal contest. European Journal of Political Economy, 14(4):587 - 603, 1998.

[Dechenaux et al., 2014] Emmanuel Dechenaux, Dan Kovenock, and Roman Sheremeta. A survey of experimental research on contests, all-pay auctions and tournaments. Experimental Economics, pages 1-61, 2014.

[DiPalantino and Vojnovic, 2009] Dominic DiPalantino and Milan Vojnovic. Crowdsourcing and all-pay auctions. In Proc. of ACM-EC, pages 119-128, 2009.

[Faravelli, 2011] Marco Faravelli. The important thing is not (always) winning but taking part: Funding public goods with contests. Journal of Public Economic Theory, 13(1):1-22, 2011.

[Fu and Lu, 2012] Qiang Fu and Jingfeng Lu. The optimal multi-stage contest. Journal of Economic Theory, 51(2):351-382, 2012.

[Galton, 1902] Francis Galton. The most suitable proportion between the value of first and second prizes. Biometrika, 1(4):385-399, 1902.

[Ghosh and Hummel, 2012] Arpita Ghosh and Patrick Hummel. Implementing optimal outcomes in social computing: A game-theoretic approach. In Proc. of $W W W$, pages 539548, 2012.
[Ghosh and Kleinberg, 2016] Arpita Ghosh and Robert Kleinberg. Optimal contest design for simple agents. ACM Transactions on Economic and Computation, 4(4):22:1-22:41, 2016.

[Glazer and Hassin, 1988] A. Glazer and R. Hassin. Optimal contests. Economic Inquiry, 26(1):133-143, 1988.

[Hazon et al., 2008] Noam Hazon, Paul E Dunne, Sarit Kraus, and Michael Wooldridge. How to rig elections and competitions. Proceedings of COMSOC, 2008.

[Jian et al., 2016] Lian Jian, Zheng Li, and Tracy Xiao Liu. Simultaneous versus sequential all-pay auctions: an experimental study. Experimental Economics, pages 1-22, 2016.

[Lev et al., 2013] Omer Lev, Maria Polukarov, Yoram Bachrach, and Jeffrey S. Rosenschein. Mergers and collusion in all-pay auctions and crowdsourcing contests. In Proc. of AAMAS, pages 675-682, 2013.

[Liu et al., 2013] Bin Liu, Jingfeng Lu, Ruqu Wang, and Jun Zhang. Prize and punishment: Optimal contest design with incomplete information. National University of Signapore Typescript, 2013.

[Liu et al., 2014] Tracy Xiao Liu, Jiang Yang, Lada A. Adamic, and Yan Chen. Crowdsourcing with all-pay auctions: A field experiment on taskcn. Management Science, 60(8):2020-2037, 2014.

[Luo et al., 2014] Tie Luo, Salil S. Kanhere, and Hwee-Pink Tan. Optimal prizes for all-pay contests in heterogeneous crowdsourcing. In Proc. of MASS, pages 136-144, 2014.

[McMillan and Rothschild, 1994] John McMillan and Michael Rothschild. Search. In Handbook of Game Theory with Economic Applications, volume 2, pages 905 - 927, 1994.

[Moldovanu and Sela, 2001] Benny Moldovanu and Aner Sela. The optimal allocation of prizes in contests. American Economic Review, 91(3):542-558, 2001.

[Moldovanu and Sela, 2006] Benny Moldovanu and Aner Sela. Contest architecture. Journal of Economic Theory, 126(1):70-96, 2006.

[Morgan, 2003] John Morgan. Sequential contests. Public Choice, 116(1):1-18, 2003.

[Nti, 1999] Kofi O. Nti. Rent-seeking with asymmetric valuations. Public Choice, 98(3):415-430, 1999.

[Rosen, 1986] Sherwin Rosen. Prizes and incentives in elimination tournaments. The American Economic Review, 76(4):701-715, 1986.

[Schlagwein and Bjorn-Andersen, 2014] Daniel Schlagwein and Niels Bjorn-Andersen. Organizational learning with crowdsourcing: The revelatory case of lego. J. of the Association of Information Systems, 15(11):754-778, 2014.

[Segev and Sela, 2014] Ella Segev and Aner Sela. Multistage sequential all-pay auctions. European Economic Review, 70:371-382, 2014.

[Stracke, 2013] Rudi Stracke. Contest design and heterogeneity. Economics Letters, 121(1):4-7, 2013. 